\title{
Aromatic ionene topology and counterion-tuned gelation of acidic aqueous solutions $\uparrow$
}

\author{
Jürgen Bachl, ${ }^{a}$ Oscar Bertran, ${ }^{b}$ Judith Mayr, ${ }^{a}$ Carlos Alemán*c,d and David Díaz Díaz ${ }^{* a, e}$ \\ Received (in $X X X, X X X) X$ th $X X X X X X X X X 20 X X$, Accepted Xth $X X X X X X X X X 20 X X$ \\ ${ }_{5}$ DOI: 10.1039/b000000x
}

Unusual gelation of acidic solutions was achieved using polycations bearing quaternary ammonium moieties. These ionene polymers are based on a disubstituted phenylene dibenzamide core, which allows the construction of different topomers (i.e. ortho-1, meta-2 and para-3). The topology of the polymers was found to play a key role on their aggregation behaviour both in pure water and in a variety of aqueous

10 acidic solutions leading to the formation of stable acidic gels. Specifically, ortho-1 showed superior gelation ability than the analogues meta-2 and para-3 in numerous solutions of different $\mathrm{pH}$ and ionic strengths. Lower critical gelation concentrations, higher gel-to-sol transition temperatures and faster gelation were usually observed for ortho-1 regardless the solvent system. Detailed computational molecular dynamic simulations revealed a major role of the counterion $\left(\mathrm{Cl}^{-}\right)$and specific

15 polymer $\cdots$ polymer interactions. In particular, hydrogen bonds, $\mathrm{N}-\mathrm{H} \cdots \pi$ interactions and intramolecular $\pi-\pi$ stacking networks are distinctive in ortho-1. In addition, counterions located at internal hydration regions also affect to such polymer $\cdots$ polymer interactions, acting as binders and, therefore, providing additional stability.

\section{Introduction}

20 A large number of important biological macromolecules (e.g. nucleic acids, polypeptides and polysaccharides) and well-known synthetic macromolecules (e.g. polyacrylic acid and poly(styrene sulfonate)) are highly charged polymers, so-called polyelectrolytes, that have been object of abundant theoretical 25 and experimental investigations during the last decades. ${ }^{1-3}$ Commercial applications of these polymers range from colloidal stabilization to superabsorbent gels, where hydrophobic, charge transfer and long-ranged electrostatic interactions mediated by mobile ions and water molecules define the physical properties 30 and, hence, the functionality of these systems.

Among different types of polyelectrolytes, polycations bearing quaternary ammonium in the backbone constitute a very interesting subgroup known as ionenes, ${ }^{4-10}$ which are usually

\footnotetext{
${ }^{a}$ Institut für Organische Chemie, Universität Regensburg, Universitätsstr. 31, 93053 Regensburg, Germany. E-mail: David.Diaz@chemie.uni-regensburg.de; Fax: + 49941 9434121; Tel.: + 499419434373

${ }^{b}$ Departament de Física Aplicada, EEI, Universitat Politècnica de Catalunya, Av. Pla de la Massa, 8, 08700 Igualada, Spain

${ }^{c}$ Departament d'Enginyeria Química, EEBE, Universitat Politècnica de Catalunya, C/ Eduard Maristany, 10-14, 08019, Barcelona, Spain

${ }^{d}$ Barcelona Research Center for Multiscale Science and Engineering, Universitat Politècnica de Catalunya C/ Eduard Maristany, 10-14, 08019, Barcelona, Spain , ${ }^{e} \quad$ IQACCSIC, Jordi Girona 18-26, 08034 Barcelona, Spain

$\dagger$ Electronic Supplementary Information (ESI) available: Additional microscopy images, rheological plots, tabular data, FTIR spectra, additional computational information. See DOI: 10.1039/b000000x/
}

prepared either by step polymerization via Menshutkin reaction 35 or cationic functionalization of precursor polymers. ${ }^{11-14}$ These polymers have been widely used as antibacterial agents, building blocks for the preparation of chromatography stationary phases, symplexes or functional gels, among other applications. ${ }^{4,15,16}$ The charge distribution, the type of counterions, the molecular weight, 40 structural flexibility and H-bonding capability are, among others, critical aspects that may influence the dynamics of the ionic chains that govern their properties. ${ }^{17-19}$ Unfortunately, a low control on some of these features as well as the lack of theoretical models developed at the atomistic level ${ }^{20-24}$ make difficult to 45 understand, predict and control their aggregation in different aqueous media. ${ }^{20}$ Within this context, we have recently reported ${ }^{25}$ a combined computational-experimental approach that led to the improvement of the hydrogelation ability of important 1,4diazabicyclo[2.2.2] octane (DABCO)-based ionenes ${ }^{26-27}$ 1-3 50 containing different structural isomers of phenylenediamine as molecular core (Figure 1). Molecular dynamics simulations of these ionenes with explicit water molecules pointed out remarkable differences in the assembly of the polymeric chains in each case. Specifically, interchain regions with high degree of 55 hydration (i.e. polymer*wwater interactions) and regions dominated by polymer $\cdots$ polymer interactions were evident in the case of ortho- (1) and meta- (2) isomeric ionenes, while domains governed by polymer $\cdots$ polymer interactions were nearly inexistent in 3. This was experimentally correlated with the 60 superior properties of the gels prepared from $\mathbf{1}^{25}$ 


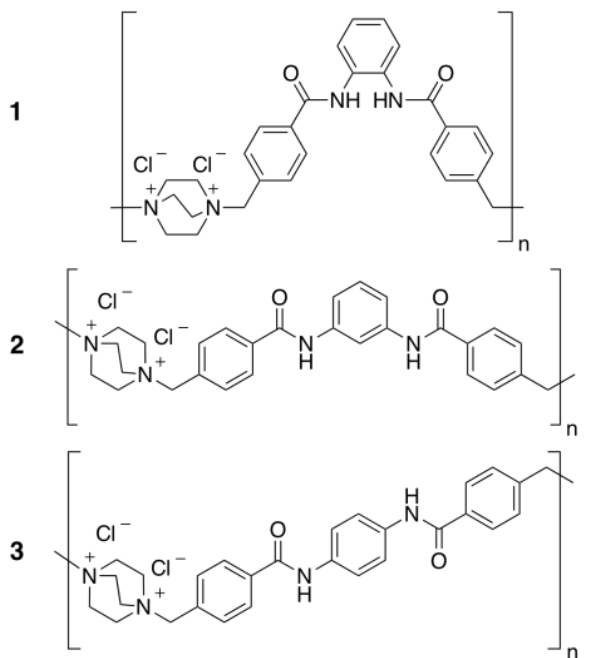

Fig. 1 Structures of topologically different DABCO-containing ionene polymers 1-3.

Despite the potential interest of gelation of acidic solutions in 5 areas such as safety and environmental remediation, ${ }^{28-30}$ this phenomenon is scarcely described in the literature and mainly associated to low molecular weight gelators. ${ }^{31-35}$ A reason behind this scenario may be the existence of labile structural moieties in many gelating agents and their potential cleavage and/or 10 undesired protonation events in acidic media. Yoshida and coworkers $^{26}$ reported that a polymer based on a $N, N^{\prime}-(p-$ phenylene)dibenzamide linkage bearing a $\mathrm{C}_{6}$-flexible spacer was able to gel a $0.1 \mathrm{M} \mathrm{HCl}$ solution, although no further studies were carried out related to this observation. To our delight, we have 15 found that the well-defined topology of ionenes 1-3 not only govern their aggregation behaviour in pure water but also in a variety of aqueous acidic solutions leading to the formation of a variety of stable acidic gels. Herein, we describe the details of this exceptional phenomenon and the understanding of the effects 20 of the different polymer topologies through computational molecular dynamic simulations.

\section{Experimental}

\section{Materials}

Unless otherwise stated all chemicals, reagents and solvents were 25 of analytical grade, purchased from commercial sources (TCI Europe, Sigma-Aldrich) and used as received without further purification. Water was bidistilled prior usage. The ionene polymers were prepared as we previously reported. ${ }^{25}$ Briefly, amidation of isomeric ortho-, meta- and para-phenylenediamine 30 with 4-(chloromethyl)benzoyl chloride in the presence of $\mathrm{Et}_{3} \mathrm{~N}$ in $\mathrm{CH}_{2} \mathrm{Cl}_{2}$ afforded the corresponding bis-benzamides in good yields (87-96\%) upon recrystallization from $\mathrm{CH}_{2} \mathrm{Cl}_{2} /$ pentane. Subsequent step-growth copolymerization with 1,4diazabicyclo[2.2.2] octane (DABCO) under equimolar conditions 35 in DMF at $80{ }^{\circ} \mathrm{C}$ gave the desired polycations $\mathbf{1}-\mathbf{3}$ as precipitates within 48-72 h in good yields (69-98\%) after filtration, washing with DMF, $\mathrm{CH}_{3} \mathrm{CN}$ and $\mathrm{CH}_{2} \mathrm{Cl}_{2}$ and drying under vacuum.

\section{Characterization methods}

FTIR spectra were recorded using a Diamond ATR (attenuated
40 total reflection) accessory (Golden Gate), in a VARIAN 1000 FTIR (Scimitar ${ }^{\text {TM }}$ Series).

Oscillatory rheology was performed with an AR 2000 Advanced rheometer (TA Instruments) equipped with a Julabo C cooling system. A $1000 \mu \mathrm{m}$ gap setting and a torque setting of ${ }_{45} 40000$ dynes $\mathrm{cm}^{-2}$ at $25^{\circ} \mathrm{C}$ were used for the measurements in a plain-plate $(20 \mathrm{~mm}$, stainless steel). The data were found to be highly reproducible for independent batches. The following experiments were carried out for each sample, using ca. $2 \mathrm{~mL}$ total gel volume: a) Dynamic Strain Sweep (DSS): variation of $G^{\prime}$ 50 and $G^{\prime \prime}$ with strain (from 0.01 to $100 \%$ ); b) Dynamic Frequency Sweep (DFS): variation of $G^{\prime}$ and $G^{\prime \prime}$ with frequency (from 0.1 to $10 \mathrm{~Hz}$ at $0.1 \%$ strain); c) Dynamic Time Sweep (DTS): variation of $G^{\prime}$ and $G^{\prime \prime}$ with time keeping the strain and frequency values constant and within the linear viscoelastic regime ( $\operatorname{strain}=0.1 \%$ 55 strain; frequency $=1 \mathrm{~Hz}$ ). Mechanical inertial effects of the measuring head were accounted by the software package to accurately evaluate the thixotropic nature of the materials through loop tests. For this, fixed rest time after sample loading and preshearing to equilibrium at different shear rates were routinely 60 made in order to minimize prehistory effects. Low frequencydependence of the moduli is characteristic of viscoelastic gels. Loop-tests involved the following steps: (1) Application of a low stress phase for $20 \mathrm{~min}$ at $0.1 \%$ oscillatory strain and $1 \mathrm{~Hz}$ frequency as defined by DTS experiments (gel state, $G^{\prime}>G^{\prime \prime}$ ), (2) ${ }_{65}$ increase of the shear strain rate until $100 \%$ strain and $1 \mathrm{~Hz}$ frequency for 2 min to ensure gel-to-sol transition $\left(G^{\prime}<G^{\prime \prime}\right)$ and minimize inertial effects, and (3) relaxation for at least $20 \mathrm{~min}$ at the same conditions as for step (1) (recovered gel state, $G^{\prime}>G^{\prime \prime}$ ). Steps (2) and (3) have been performed twice to ensure the 70 reversible nature of the phase transitions.

Electron microscopy of the samples was conducted with the following instruments: (1) JEOL-2000 FXII transmission electron microscope $($ TEM, resolution $=0.28 \mathrm{~nm})$ equipped with a CCD Gatan 694 digital camera and operating at $10 \mathrm{kV}$ (accelerating 75 voltage). (2) Carl Zeiss Merlin field emission scanning electron microscope (FESEM, resolution $0.8 \mathrm{~nm}$ ) equipped with a digital camera and operating at $5 \mathrm{kV}$ (accelerating voltage) and $10 \mu \mathrm{A}$ (emission current). Preparation of the samples for TEM: $10 \mu \mathrm{L}$ of the gel suspension was allowed to adsorb for $30 \mathrm{~s}$ onto carbon80 coated grids (300 meshes, from TED PELLA, Inc.). After the adsorption, the excess solvent was removed by touching the edges with a small piece of filter paper (Whatman). The specimens were then dried overnight in a desiccator at low pressure and room temperature (RT). Patches of the gel were first 85 searched to be sure that the observed structures originate from the gel. Micrographs were taken from structures at the periphery of the patches where the fibers were deposited in a layer thin enough to be observed by TEM. Preparation of the samples for FESEM: Samples of the cryogels were prepared by the freeze-drying (FD) 90 method: ${ }^{36}$ An Eppendorf tube containing the corresponding gel $(100-200 \mu \mathrm{L})$ was frozen in liquid nitrogen or dry ice/acetone and the sample was immediately evaporated under reduced pressure $(0.6 \mathrm{mmHg})$ for 2 days at RT. A fibrous solid was obtained, which was placed on top of a tin plate and shielded by ${ }_{95} \mathrm{Pt}$ (40 mA during $30 \mathrm{~s}$; film thickness $\approx 5 \mathrm{~nm}$ ).

Polarized optical microscopy (POM) was conducted using a Olympus BX51 microscope equipped with a Olympus DP12 


\section{Cite this: DOI: $10.1039 / \mathrm{c0xx} 00000 \mathrm{x}$}

\section{www.rsc.org/xxxxxx}

ARTICLE TYPE

digital camera at cross polarization of $90^{\circ}$ using polarization filters. The samples were sandwiched between two cover glass slides for observation.

Elemental analyses were recorded on a Heraeus CHN 5 elemental analyzer.

In order to achieve adequate solubility and mobility of the polymers for gel permeation chromatography (GPC) analysis, it was necessary to carry out anion exchange of chloride by bis(trifluoromethanesulfonyl)amide (TFSA) anions using lithium 10 bis(trifluoromethanesulfonyl)azanide in hot water as previously described. $^{25}$ The main physical properties of the polymers $\left(M_{\mathrm{n}}\right.$, $\left.M_{\mathrm{w}}, n, Ð\right)$ were previously determined and can be found in the Supporting Information.

\section{Preparation of gel materials}

15 Typically, a weighed amount of the corresponding ionene polymer and $0.5 \mathrm{~mL}$ of the appropriate aqueous medium were placed into a screw-capped glass vial $(4 \mathrm{~cm}$ length and $1 \mathrm{~cm}$ diameter) and gently heated with a heat-gun until the solid material was completely dissolved. The resulting isotropic 20 solution was then spontaneously cooled down to RT. No control over temperature rate during the heating-cooling process was applied. The material was preliminarily classified as "gel" if it did not exhibit gravitational flow upon turning the vial upsidedown at RT. The state was further confirmed by rheological 25 measurements.

\section{Determination of critical gelation concentration (CGC)}

CGC values were estimated by continuously adding aliquots of solvent $(0.02-0.1 \mathrm{~mL})$ into vials containing the corresponding ionene polymer and performing a typical heating-cooling 30 protocol for gel-formation until no gelation was observed. The starting point for CGC determinations was $200 \mathrm{~g} \mathrm{~L}^{-1}$. No further experiments were made if gelation was not achieved at $500 \mathrm{~g} \mathrm{~L}^{-1}$. Non-gel containing vials (i.e. clear solution after 10 days) were frequently left at RT and visually monitored for possible 35 gelation/crystallization over time.

Determinations of thermal gel-to-sol transition temperature $\left(T_{g e l}\right)$

$T_{\text {gel }}$ values were determined by the inverse flow method (the seal vial containing the hydrogel was hung horizontally into an oil 40 bath, which was heated up at $\left.2{ }^{\circ} \mathrm{C} \mathrm{min}^{-1}\right) .{ }^{37}$ Herein, the temperature at which the gel started to break was defined as $T_{\text {gel }}$. Each measurement was made by duplicate and the average values reported.

\section{Determination of $\mathrm{HCl}$ gas absorption}

${ }_{45}$ The desired absorbing material was placed into a $50 \mathrm{~mL}$ Schlenk flask and exposed to a continuous flow of $\mathrm{HCl}$ gas generated in situ from the addition of concentrated $\mathrm{H}_{2} \mathrm{SO}_{4}$ via a dropping funnel over solid $\mathrm{NaCl}$ placed in a separate flask with a gas exit connected to the flask containing the polymeric material (Figure
${ }_{50} \mathrm{~S} 1$ ). The absorbed amount of $\mathrm{HCl}$ was subsequently determined by titration of the material with $0.1 \mathrm{M} \mathrm{NaOH}$.

\section{Computational studies}

Production NPT Molecular Dynamics (MD) simulations were performed for 1, $\mathbf{2}$ and $\mathbf{3}$ hydrogels. For each system, a model ${ }_{55}$ containing six polymer chains with six repeat units each one (adding a two chloride counterion per repeat unit), $1000 \mathrm{H}_{3} \mathrm{O}^{+} \cdot \mathrm{Cl}^{-}$ ion pairs and around $145 \mathrm{~K}$ water molecules were considered. For the sake of completeness, $\mathrm{MD}$ simulations of $\mathbf{1}$ in aqueous solution (i.e. replacing the $1000 \mathrm{H}_{3} \mathrm{O}^{+} \cdot \mathrm{Cl}^{-}$ion pairs by 1000 water 60 molecules) were also run, the resulting system being denoted 1HCl(free). The total number of explicit particles for each production trajectory, which were $45 \mathrm{~ns}$ long, was around $450 \mathrm{~K}$. Details about the computational methods, equilibration protocols and composition of each system are provided in the Supporting ${ }_{65}$ Information. All radial distribution functions were calculated considering the last $25 \mathrm{~ns}$ of the production runs. The gelation capacity of 1-3 and 1-free $(\mathbf{H C l})$ was evaluated by quantifying the dimensions of the hydration regions through the box-counting method applied to a regular three-dimensional grid. ${ }^{38}$

\section{${ }_{70}$ Results and discussion}

\section{Gelation of aqueous acidic solutions}

During the study of the gelation ability of ionenes $\mathbf{1}-\mathbf{3}$ we found that $\mathbf{1}$ (ortho-isomer) and $\mathbf{3}$ (para-isomer) efficiently gelled $\mathrm{HCl}$ solutions $(0.1 \mathrm{M}, \mathrm{pH}=1.0)$ at their corresponding critical 75 gelation concentration (CGC) determined in pure water $\left(25 \mathrm{~g} \mathrm{~L}^{-1}\right.$ and $48 \mathrm{~g} \mathrm{~L}^{-1}$, respectively). In contrast, meta-isomer 2 failed to gel these $\mathrm{HCl}$ solutions even at 2-fold higher gelator concentration (up to $200 \mathrm{~g} \mathrm{~L}^{-1}$ ) than that in pure water. As shown in Table 1, further investigations revealed that compounds $\mathbf{1}$ and ${ }_{80} 3$ were also able to promote effective gelation of $0.1 \mathrm{M} \mathrm{HCl}$ solutions not only at concentrations even lower than the CGC established in pure water, but also with much faster kinetics ( $c a$. 100-times and 50-times shorter gelation times than in water for 1 and $\mathbf{3}$, respectively). Motivated by these results, we decided to 85 explore the gelation ability of these systems in other acidic solutions. Ionene 1 formed stable and homogeneous gels within $30 \mathrm{~min}$ in $\mathrm{HBr}, \mathrm{HI}, \mathrm{H}_{3} \mathrm{PO}_{4}$ and $\mathrm{HNO}_{3}$ solutions $(0.1 \mathrm{M}, \mathrm{pH}=1.0$ for $\mathrm{HBr}, \mathrm{HI}$, and $\left.\mathrm{HNO}_{3}, \mathrm{pH}=1.62 \mathrm{H}_{3} \mathrm{PO}_{4}\right)$, whereas the gelation ability of $\mathbf{2}$ and $\mathbf{3}$ was rather limited and several of their gels were 90 not homogeneous and underwent gradual water loss over time (Table 1 and Figure S2). No gelation was observed in $\mathrm{H}_{2} \mathrm{SO}_{4}(0.1$ $\mathrm{M}, \mathrm{pH}=0.96)$ for any of the ionenes, which led to turbid solutions at concentrations ranging between $10 \mathrm{~g} \mathrm{~L}^{-1}$ and $200 \mathrm{~g} \mathrm{~L}^{-}$ 1. Remarkably, $\mathbf{1}$ and $\mathbf{3}$ also showed the capacity to form stable 95 gels in solutions of organic acids such as acetic acid $\left(\mathrm{CH}_{3} \mathrm{COOH}\right.$, $0.1 \mathrm{M}, \mathrm{pH}=2.87)$ and formic acid $(\mathrm{HCOOH}, 0.1 \mathrm{M}, \mathrm{pH}=2.37)$, being 1 the most efficient gelator in terms of gelation concentration, kinetics and/or thermal stability of the gels. In 
terms of optical appearance, and in contrast to the hydrogel obtained from 1 in water, all gels described in Table 1 were opaque except the one made of $\mathbf{1}$ in acetic acid, which was translucent. These appearances suggested the formation of 5 aggregates in acidic solutions bigger than the wavelength of visible light $(350-750 \mathrm{~nm})$ causing its scattering (vide infra, Figure 3D). The acidic gels prepared from 1 were stable for several months at room temperature without detriment of their consistency and visual appearance.

10 At this point we decided to take $\mathrm{HCl}$ solution as a model acidic medium to investigate and compare other properties of the obtained gels compared to those prepared in pure water. An important effect of the $\mathrm{Cl}^{-}$counterion was revealed by the ability of $\mathbf{1}$ to gel also solutions of different ionic strengths containing 15 inorganic chloride salts such as $\mathrm{NaCl}, \mathrm{CsCl}, \mathrm{NH}_{4} \mathrm{Cl}(\mathrm{pH}=5.1)$ and $\mathrm{CaCl}_{2}$, albeit the use of 3.5-4 fold excess in gelator concentration compared to the CGC in pure water was necessary to obtain the gels (Table 1). The superior gelation properties of $\mathbf{1}$ were also evident in these solutions as neither $\mathbf{2}$ nor $\mathbf{3}$ were able 20 to form gels at concentrations ranging from $10 \mathrm{~g} \mathrm{~L}^{-1}$ and $200 \mathrm{~g} \mathrm{~L}^{-}$ 1 . These results suggest an apparent synergistic effect of both $\mathrm{H}^{+}$ and $\mathrm{Cl}^{-}$ions to facilitate the gelation process using $\mathbf{1}$, thus lowering the CGC and increasing the stability of the resulting gel in comparison to pure water.

Table 1 Effect of different aqueous environments containing various acids and inorganic $\mathrm{Cl}^{-}$sources on the gelation properties of ionenes $\mathbf{1}-\mathbf{3}$ with respect to pure water. ${ }^{a}$

\begin{tabular}{|c|c|c|c|c|c|c|c|c|c|}
\hline \multirow{2}{*}{$\begin{array}{l}\text { Aqueous } \\
\text { medium }^{b}\end{array}$} & \multicolumn{3}{|c|}{$o$-Ionene 1} & \multicolumn{3}{|c|}{$m$-Ionene $\mathbf{2}$} & \multicolumn{3}{|c|}{$p$-Ionene $\mathbf{3}$} \\
\hline & $\begin{array}{c}\mathrm{CGC} \\
\left(\mathrm{g} \mathrm{L}^{-1}\right)\end{array}$ & $\begin{array}{l}\text { Gel-time } \\
(\mathrm{min})\end{array}$ & $\begin{array}{l}T_{\text {gel }} \\
\left({ }^{\circ} \mathrm{C}\right)\end{array}$ & $\begin{array}{c}\mathrm{CGC} \\
\left(\mathrm{g} \mathrm{L}^{-1}\right)\end{array}$ & $\begin{array}{l}\text { Gel-time } \\
(\min )\end{array}$ & $\begin{array}{l}T_{\text {gel }} \\
\left({ }^{\circ} \mathrm{C}\right)\end{array}$ & $\begin{array}{c}\mathrm{CGC} \\
\left(\mathrm{g} \mathrm{L}^{-1}\right)\end{array}$ & $\begin{array}{l}\text { Gel-time } \\
(\min )\end{array}$ & $\begin{array}{l}T_{\text {gel }} \\
\left({ }^{\circ} \mathrm{C}\right)\end{array}$ \\
\hline $\mathrm{H}_{2} \mathrm{O}$ & $25 \pm 2$ & $1080 \pm 60$ & $56 \pm 1$ & $100 \pm 10$ & $1260 \pm 40$ & $59 \pm 1$ & $48 \pm 5$ & $2640 \pm 240$ & $49 \pm 1$ \\
\hline $\mathrm{HCl}$ & $22 \pm 2$ & $10 \pm 2$ & $60 \pm 2$ & S & - & - & $33 \pm 3$ & $50 \pm 5$ & $63 \pm 1$ \\
\hline $\mathrm{HBr}$ & $56 \pm 2$ & $11 \pm 5$ & $44 \pm 1$ & $170 \pm 30$ & $1 \pm 0.5$ & $-{ }^{d}$ & $58 \pm 3$ & $30 \pm 10$ & $34 \pm 4$ \\
\hline HI & $68 \pm 3$ & $6 \pm 1$ & $41 \pm 2$ & $59 \pm 9$ & $420 \pm 60$ & $32 \pm 2$ & I & - & - \\
\hline $\mathrm{H}_{3} \mathrm{PO}_{4}$ & $46 \pm 4$ & $20 \pm 2$ & $76 \pm 1$ & S & - & - & $105 \pm 11$ & $68 \pm 8$ & $55 \pm 2$ \\
\hline $\mathrm{HNO}_{3}$ & $46 \pm 1$ & $30 \pm 5$ & $35 \pm 1$ & $139 \pm 29$ & $4 \pm 1$ & $-{ }^{d}$ & $88 \pm 2$ & $15 \pm 1$ & $-{ }^{d}$ \\
\hline $\mathrm{H}_{2} \mathrm{SO}_{4}$ & $S$ & - & - & $S$ & - & - & S & - & - \\
\hline $\mathrm{CH}_{3} \mathrm{COOH}$ & $48 \pm 4$ & $5 \pm 1$ & $61 \pm 2$ & $S$ & - & - & $110 \pm 10$ & $30 \pm 5$ & $33 \pm 2$ \\
\hline $\mathrm{HCOOH}$ & $45 \pm 1$ & $90 \pm 20$ & $57 \pm 2$ & $150 \pm 50$ & $3 \pm 1$ & $-{ }^{d}$ & $68 \pm 1$ & $140 \pm 20$ & $67 \pm 7$ \\
\hline $\mathrm{NaCl}$ & $92 \pm 9$ & $6 \pm 1$ & $54 \pm 3$ & $\mathrm{P}$ & - & - & $P$ & - & - \\
\hline $\mathrm{CsCl}$ & $97 \pm 9$ & $3 \pm 1$ & $53 \pm 2$ & $\mathrm{P}$ & - & - & $P$ & - & - \\
\hline $\mathrm{NH}_{4} \mathrm{Cl}$ & $100 \pm 10$ & $4 \pm 1$ & $63 \pm 2$ & $\mathrm{P}$ & - & - & $P$ & - & - \\
\hline $\mathrm{CaCl}_{2}{ }^{c}$ & $89 \pm 9$ & $4 \pm 1$ & $36 \pm 2$ & $\mathrm{P}$ & - & - & $\mathrm{P}$ & - & - \\
\hline
\end{tabular}

${ }^{a}$ Experimental errors were determined from at least two randomized measurements. ${ }^{b}$ Unless otherwise indicated, the concentration of aqueous solutions was adjusted to $0.1 \mathrm{M} .{ }^{c}$ Concentration was adjusted to $0.05 \mathrm{M}$. ${ }^{\mathrm{d}}$ Gradual water loss of the material precluded an accurate determination of the $T_{\text {gel }}$. 30 Abbreviations: $\mathrm{I}=$ insoluble, $\mathrm{S}=$ solution, $\mathrm{P}=$ precipitate.

Additional positive effects of the $\mathrm{HCl}$ medium were obvious by comparing the gelation kinetics and the thermal stabilities of gels with those obtained in pure water at comparable ionene 35 concentrations (Figure 2). Thermal sol-to-gel transition temperatures $\left(T_{g e l}\right)$ of the gels prepared from $\mathbf{1}$ and $\mathbf{3}$ were found to be increased by $4{ }^{\circ} \mathrm{C}$ and $14{ }^{\circ} \mathrm{C}$, respectively, with respect to those obtained in water (Figure 2A). Furthermore, a remarkable decrease in the gelation time to about $20 \%$ of the value obtained 40 in water was visible for both compounds $\mathbf{1}$ and $\mathbf{3}$ in $\mathrm{HCl}$ solutions (Figure 2B). The optimum concentration of $\mathrm{HCl}$ in aqueous solution was determined by testing different concentrations at the CGC of the desired gelator in pure water, and comparing the effect on both the $T_{g e l}$ and the gelation kinetics. The results are 45 summarized in Table $\mathrm{S} 1$ and Figure 2 and indicate that a concentration of $\mathrm{HCl}$ of $0.1 \mathrm{M}$ promotes the most effective gelation characterized by the highest $T_{\text {gel }}$ and gelation kinetics for both ionenes. Gels made from $\mathbf{1}$ and $\mathbf{3}$ could also be prepared at acid concentrations of $1.0 \mathrm{M}$ and $0.5 \mathrm{M}$, respectively, albeit with 50 a detriment of the studied gels properties. Higher concentrations in each case lead to clear viscous solutions. In addition, and as also observed in pure water, ${ }^{25}$ both the thermal stability of the gels and the gelation kinetics in acidic medium were enhanced by increasing the ionene concentration (e.g. gel prepared from $\mathbf{1}$ in
${ }_{55} 0.1 \mathrm{M} \mathrm{HCl}$ at its CGC displayed a $T_{\text {gel }}$ of $60 \pm 2{ }^{\circ} \mathrm{C}$ and a gelation time of $10 \pm 2 \mathrm{~min}$, whereas $\mathrm{ca}$. 4-fold increase in the concentration of the ionene afforded an opaque gel within $4 \pm 0.2$ min and with a $T_{\text {gel }}$ of $72 \pm 1^{\circ} \mathrm{C}$ ) (Table $\mathrm{S} 1$ ).

It is worth mentioning that no degradation products of the ${ }_{60}$ polymers were detected in the respective acidic solutions, at least during the time scale of the reported experiments. Furthermore, in our previous work we demonstrated that the topology of these polymers plays a critical role on their hydrogelation ability, being the molecular weight much less relevant. ${ }^{25}$ The weight-average 65 molecular weight, polymerization degree and dispersity values are very similar for compounds $\mathbf{1}$ and $\mathbf{2}$, whereas these values are relatively higher for the isomer $\mathbf{3}$ (see Supporting Information). Thus, the better gelation properties (Table 1) according to the lower molecular weight of $\mathbf{1}$ in comparison to $\mathbf{3}$ seems to be a 70 more complex scenario if the isomer $\mathbf{2}$ is also taking into consideration. This is in agreement with the major influence of the topology over the molecular weight of these polymers on their self-assembly behaviour in aqueous medium and on the properties of the so-formed aggregates. 
A

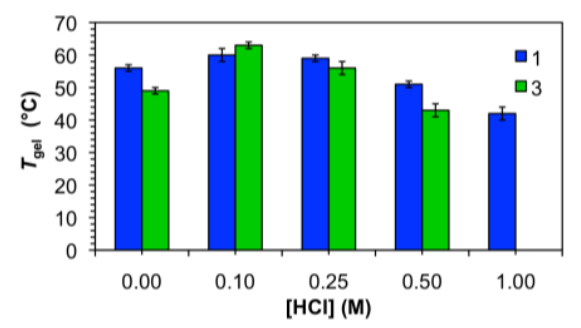

B

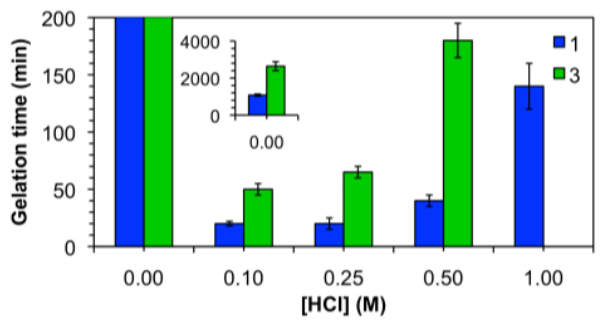

Table 2 Rheological properties of the gels made from ionenes (IN) $\mathbf{1}$ and 253 in pure water and $0.1 \mathrm{M} \mathrm{HCl}$ solution at their corresponding CGC as indicated in Table $1 .^{a}$

\begin{tabular}{c|cc|cc|cc|cc}
\hline \multirow{2}{*}{$\mathrm{IN}$} & \multicolumn{2}{|c|}{$G^{\prime}(\mathrm{Pa})$} & \multicolumn{2}{c|}{$G^{\prime \prime}(\mathrm{Pa})$} & \multicolumn{2}{c|}{$\tan \delta$} & \multicolumn{2}{c}{$\gamma(\%)$} \\
& $\mathrm{H}_{2} \mathrm{O}$ & $\mathrm{HCl}$ & $\mathrm{H}_{2} \mathrm{O}$ & $\mathrm{HCl}$ & $\mathrm{H}_{2} \mathrm{O}$ & $\mathrm{HCl}$ & $\mathrm{H}_{2} \mathrm{O}$ & $\mathrm{HCl}$ \\
\hline \multirow{2}{*}{$\mathbf{1}$} & 510 & 4646 & 72 & 634 & 0.143 & 0.136 & 15 & 4 \\
& \pm 96 & \pm 138 & \pm 2 & \pm 33 & \pm 0.016 & \pm 0.003 & \pm 7 & \pm 0.6 \\
\hdashline $\mathbf{3}$ & 218 & 1916 & 37 & 234 & 0.169 & 0.121 & 32 & 13 \\
& \pm 27 & \pm 461 & \pm 6 & \pm 74 & \pm 0.006 & \pm 0.007 & \pm 9 & \pm 0.2 \\
\hline
\end{tabular}

${ }^{a}$ Experimental errors were determined from at least two randomized measurements.

Fig. 2 Effect of $\mathrm{HCl}$ concentration on typical gelation properties (A: $T_{\mathrm{gel}}$; $\mathrm{B}$ : gelation time) of ionenes $\mathbf{1}$ and $\mathbf{3}$ at their CGC in water as indicated in Table 1.

5 Dynamic oscillatory rheology was conducted in order to confirm the viscoelastic gel nature of the materials and compare the overall mechanical stabilities towards external shear forces (Table 2 and Figure 3). Appropriate dynamic frequency sweep (DFS), dynamic strain sweep (DSS) and dynamic time sweep 10 (DTS) experiments for the gels made of $\mathbf{1}$ and $\mathbf{3}$ both in water and in $\mathrm{HCl}$ solutions revealed a remarkable enhancement of the mechanical stabilities of the gels prepared in $\mathrm{HCl}$, as indicated by higher storage modulus $G^{\prime}$ (one order of magnitude higher than in water) and lower $\tan \delta$ values (Table 2 and Figure 3A-B). On the 15 other hand, the materials derived from $\mathrm{HCl}$ seem to be more brittle in nature as indicated by the smaller maximum strain at break $(\gamma)$ of the gels. Furthermore, the macroscopic thixotropic behavior of compound $\mathbf{1}$ that we previously observed in water ${ }^{25}$ is also preserved in $0.1 \mathrm{M} \mathrm{HCl}$ solution and supported by a three20 step loop test (i.e. $>97 \%$ of the original moduli values was recovered after the second relaxation cycle) performed as described in the Experimental Section (Table 2 and Figure 3C). 


\title{
Cite this: DOI: 10.1039/c1xx00000x
}

\author{
www.rsc.org/materials
}

PAPER

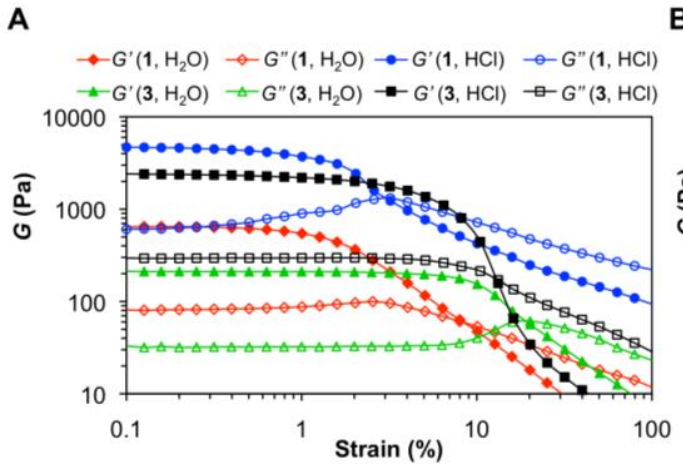

C

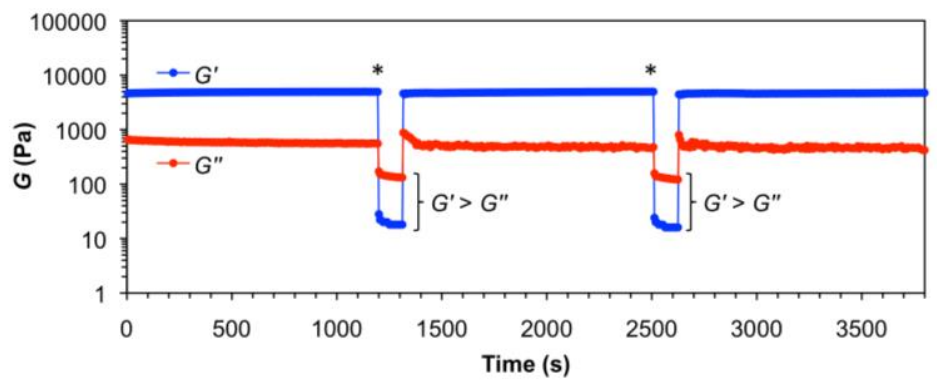

B

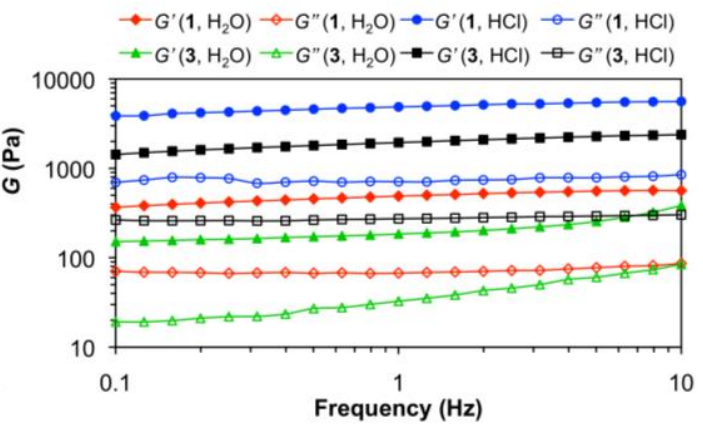

D

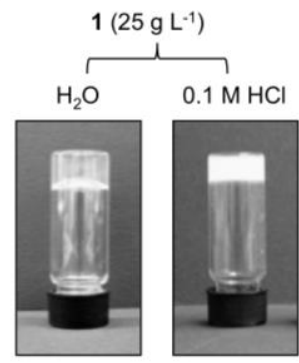

Fig. 3 A, B) Comparative oscillatory rheological measurements (DSS and DFS experiments, respectively) of the gels prepared from 1 and 3 in water and $0.1 \mathrm{M} \mathrm{HCl}$ solution at their CGC in water as indicated in Table 1. DTS plots are given in the Supporting Information (Figure S3). C) Three-step loop test showing the thixotropic behavior of the model gel made from $1\left(25 \mathrm{~g} \mathrm{~L}^{-1}\right)$ in $0.1 \mathrm{M} \mathrm{HCl}$ solution. The asterisks indicate the application of a high shear 5 strain (100\%). D) Representative digital photographs of upside-down vials containing gels derived from compound $\mathbf{1}\left(25 \mathrm{~g} \mathrm{~L}^{-1}\right)$ in pure water $($ left $)$ and 0.1 $\mathrm{M} \mathrm{HCl}$ solution (right).

Optical microscopy images of the gels made of $1\left(25 \mathrm{~g} \mathrm{~L}^{-1}\right)$ in both $0.1 \mathrm{M} \mathrm{HCl}$ and pure water revealed the presence of 10 distinctive domains of birefringence under crossed nicols dependent on the nature of the aqueous medium, indicating the formation of different anisotropic aggregates (Figure S4A-D). FESEM images of the corresponding xerogels, obtained by the freeze-drying method, ${ }^{37}$ confirmed the induction of different 15 morphologies caused by the nature of the aqueous environment. Specifically, dense and macroporous networks formed by connected leaf-like layers with interlamellar distances in a range of 5-10 $\mu \mathrm{m}$ were visualized for the xerogels of $\mathbf{1}$ and $\mathbf{3}$ in pure water (Figure S4E,F). In sharp contrast, rougher and denser 20 agglomerates could be observed for xerogels made of both ionenes in $0.1 \mathrm{M} \mathrm{HCl}$ (Figure S4G,H). Agglomerates resembling pebble stones (average diameter for individual particles $\sim 1-5$ $\mu \mathrm{m})$ were clearly characteristic for xerogels prepared from ionene 3 (Figure S4H). TEM images derived from compound $\mathbf{1}$ in pure 25 water revealed overlapped lamellar structures consisting of large and homogeneous sheets (Figure S4I), in good agreement with the findings obtained by FESEM. On the other hand, TEM images of the materials derived from acidic environment showed the presence of very dense and non-layered structures (Figure $\left.{ }_{30} \mathrm{~S} 4 \mathrm{~J}\right)$.
As expected, comparative FTIR studies using $1\left(25 \mathrm{~g} \mathrm{~L}^{-1}\right)$ suggested the involvement of amide hydrogen bonds and aromatic interactions during the gelation process in both pure water and $\mathrm{HCl}$ solution (Figure S5). It is worth mentioning that 35 the ionene hydrogel in $\mathrm{HCl}$ solution showed a slight shift of both $\mathrm{CO}$ and $\mathrm{NH}$ resonances to lower energy with respect to the solid ionene (e.g. from ca. $1646 \mathrm{~cm}^{-1}$ to $1635 \mathrm{~cm}^{-1}$ for amide I bands, and from $c a .3290 \mathrm{~cm}^{-1}$ to $3255 \mathrm{~cm}^{-1}$ for $\mathrm{NH}$ stretching bands). Nevertheless, such minor differences disappeared in the case of 40 pure water, suggesting that the above interactions are also present in the solid state of $\mathbf{1}$. Moreover, solid ionene $\mathbf{1}$ also showed affinity by in situ generated $\mathrm{HCl}$ gas (i.e. $500 \mathrm{mg}$ of solid 1 absorbed $203 \mathrm{mg}$ of $\mathrm{HCl}$ gas within $1.5 \mathrm{~h}$ ) as demonstrated by titration experiments. At this point, we performed computational 45 studies in order to provide a reasonable explanation for the effects of hydrochloric acid on the gelation process of the different ionenes as well as on the properties of the corresponding gels.

\section{Computational studies}

Simulations of ionenes 1-3 were performed considering an 50 aqueous solution containing $1000 \mathrm{H}_{3} \mathrm{O}^{+} \cdot \mathrm{Cl}^{-}$ion pairs to provide microscopic information about the relationships between the substitution pattern of the phenyl ring and the gelation capacity in 
acidic solutions. Visual inspection of the recorded snapshots reveals important differences between the three systems (Figure $4 \mathrm{~A})$. First, the degree of assembly among the polymer chains is different for the three ionenes. Thus, polymer $\cdots$ polymer 5 interactions dominate on $\mathbf{1}$ while the dimensions of the internal hydration areas progressively increase for $\mathbf{2}$ and, especially, $\mathbf{3}$. Indeed, polymer $\cdots$ polymer interactions are mainly localized at the central region for the latter. Thus, $\mathbf{1}$ dominates with respect to the other two systems not only in terms of specific interactions 10 (e.g. hydrogen bonds, $\mathrm{N}-\mathrm{H} \cdots \pi$ and $\pi \cdots \pi$ ), a proved below, but also in van der Waals forces (i.e. steric contacts among the polymer chains). Although details about differences in the nature of polymer $\cdots$ polymer interactions are provided below, differences among the three systems have been mesoscopically 15 quantified by applying the box-counting method.

For this purpose, simulation boxes of $\mathbf{1}-\mathbf{3}$ have been divided into cubes of side length $\ell$ and $M(\ell)$ against $\ell$ represented in a double-logarithmic plot, where $M(\ell)$ is the number of cubes of side length $\ell$ that contain at least one polymer atom. Results, 20 which are displayed in Figure 4B, evidence an almost perfect straight line for $\mathbf{1}, \mathbf{2}$ and $\mathbf{3}$ when $\ell \leq 22,14$ and $13 \AA$, respectively, while $M(\ell)$ follows a disperse behaviour for higher $\ell$ values. It is worth noting that this threshold $\ell$ value decreases with the increasing size of the hydration regions, corroborating 25 that the gelation capacity varies as follows: $\mathbf{1}>\mathbf{2}>\mathbf{3}$. Thus, the threshold $\ell$ value decreases when the range of length scales in which the structural self-similarity (i.e. similar features in terms of molecular organization) increases. For comparison, additional MD trajectories were run for $\mathbf{1 - f r e e}(\mathbf{H C l})$. Analysis of the results, 30 which are included in Figure 4B, reflects significant differences between 1 and $\mathbf{1 - f r e e}(\mathbf{H C l})$. Thus, the latter exhibits behaviour similar to 2 with a straight line for $\ell \leq 14 \AA$. These results demonstrate that the gelation process not only depends on the polymer topology but also on the $\mathrm{pH}$ of the aqueous solution. ${ }_{35}$ Thus, results displayed in Figure $4 \mathrm{~B}$ points out that the incorporation of $\mathrm{HCl}$ favours the gelation process of the orthoisomer decreasing the size of the hydration regions.

Experimental observations discussed in the previous section proved that, in acid aqueous solution, the gelation capacity of the 40 investigated ionenes depends on the topological constraints induced by the substitution pattern of the aromatic ring. These topological constraints are expected to influence the nature of the environment...polymer and polymer...polymer interactions, which obviously play a fundamental role in the gelation ${ }_{45}$ phenomenon. Regarding to environment $\cdots$ polymer interactions, inspection of the radial distribution functions for $\mathrm{Cl}^{-} \ldots{ }^{+} \mathrm{N}\left(g_{\mathrm{Cl}-\mathrm{N}}\right)$ and $\mathrm{Cl}^{-} \cdots \mathrm{X}_{\mathrm{cm}}\left(g_{\mathrm{Cl}-\mathrm{X}}\right)$ pairs, where $\mathrm{X}_{\mathrm{cm}}$ refers to the centre of masses of the aromatic rings, reveals that the amount of $\mathrm{Cl}^{-}$ anions surrounding or being entrapped by the polymer chains (i.e. 50 at a distance $r \leq 8 \AA$ ) decreases as follows: $\mathbf{1}>\mathbf{2}>\mathbf{3}$ (Figure 4C).

Moreover, detailed analyses of the residence times $(\tau)$ reveal that the molecular architecture affects the stability of these interactions. Thus, the number of $\mathrm{Cl}^{-}$at $r \leq 8 \AA$ from polymer chains having $\tau>1.5 \mathrm{~ns}$ is 32,25 and 12 for $\mathbf{1 , 2}$ and $\mathbf{3}$, ${ }_{55}$ respectively.
A

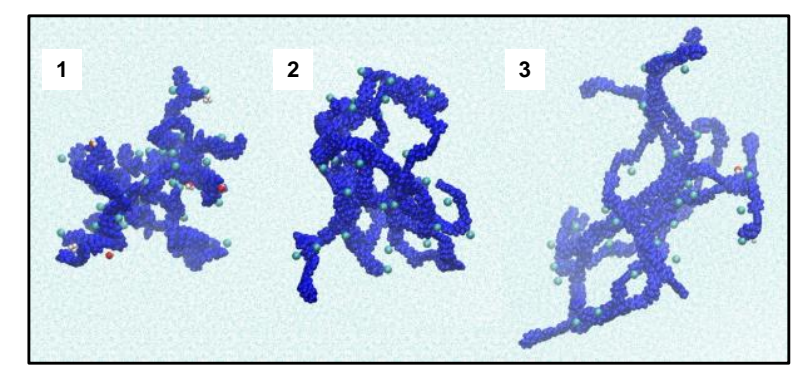

B
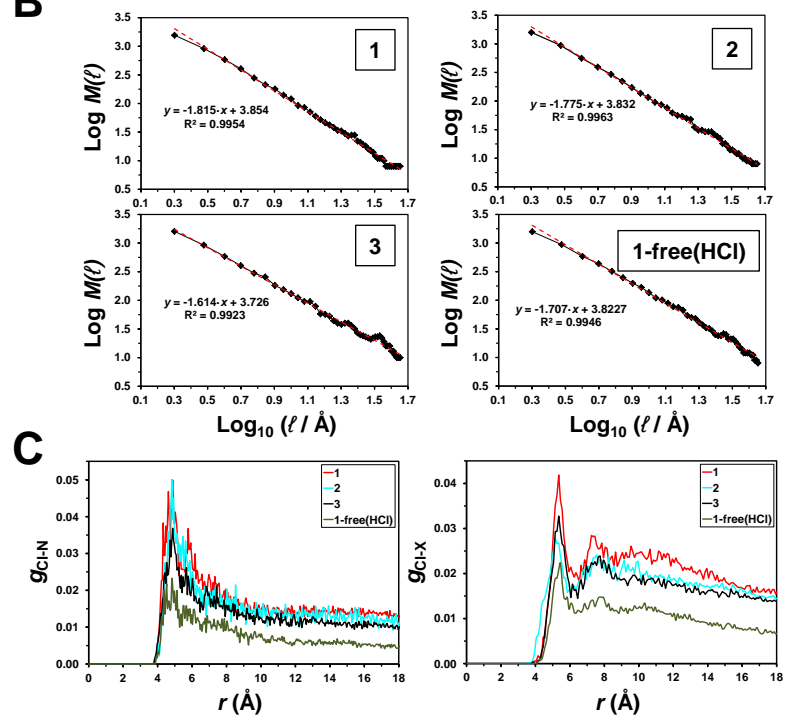

Fig. 4 A) Last snapshot of the MD simulations performed for 1-3. B) Self-similarity study based on the counting-box method for 1-3 and 1${ }_{60}$ free(HCl). $M(\ell)$ corresponds to the number of cubes of side length $\ell$, in which the simulation box was divided, that contain at least one polymer atom. C) Radial distribution functions for $\mathrm{Cl}^{-} \cdots{ }^{+} \mathrm{N}\left(g_{\mathrm{Cl}-\mathrm{N}}\right)$ and $\mathrm{Cl}^{-} \cdots \mathrm{X}_{\mathrm{cm}}$ $\left(g_{\mathrm{Cl}-\mathrm{X}}\right)$ pairs. $\mathrm{X}_{\mathrm{cm}}$ refers to the center of masses of the aromatic rings. For comparison with the radial distribution functions calculated for $\mathbf{1 - 3}$, the 65 profiles obtained for $\mathbf{1}$-free $(\mathbf{H C l})$ were scaled $(\times 6.7)$.

Similarly, the ortho, meta and para topologies of the $N, N^{\prime}$ (phenylene)dibenzamide linkages influence the strength of $\mathrm{Cl}^{-}$ ... polymer interactions. Specifically, the $g_{\mathrm{Cl}-\mathrm{N}}$ profile calculated for 1 shows two very narrow and sharp peaks at $r<4.82 \AA$, 70 which are not detected for $\mathbf{2}$ and $\mathbf{3}$. Accordingly, the electrostatic interaction between the $\mathrm{Cl}^{-}$and the positively charged groups of the polymer chains are stronger for $\mathbf{1}$ than for $\mathbf{2}$ and $\mathbf{3}$. On the other hand, the $g_{\mathrm{Cl}-\mathrm{N}}$ and $g_{\mathrm{Cl}-\mathrm{X}}$ profiles calculated for $\mathbf{1 - f r e e}(\mathbf{H C l})$, which are included in Figure $4 \mathrm{C}$, were scaled $(\times 6.7)$ for 75 comparison with those obtained for $\mathbf{1 - 3}$ since the number of $\mathrm{Cl}^{-}$ anions in the simulation box is significantly smaller than for the former than for latters (i.e. 72 counterions vs. 72 counteriones + 1000 anions from $\mathrm{H}_{3} \mathrm{O}^{+} \cdot \mathrm{Cl}^{-}$pairs). Inspection of the position of the peaks reveals that the strength of the $\mathrm{Cl}^{-} \ldots{ }^{+} \mathrm{N}$ electrostatic 80 interactions is similar in $\mathbf{1 - f r e e}(\mathbf{H C l})$ than in $\mathbf{1}$, albeit their abundance is practically negligible in the former.

The $g_{\mathrm{Cl}-\mathrm{X}}$ profiles evidence that $\mathrm{Cl}^{-}$anions are closer to the centre of the aromatic rings for $\mathbf{2}$ than for $\mathbf{1}$ and $\mathbf{3}$. This phenomenon is expected to produce some repulsive interactions ${ }_{85}$ between the $\mathrm{Cl}^{-}$anions and the aromatic rings of $\mathbf{2}$, affecting also 
the attractive $\mathrm{Cl}^{-} \ldots{ }^{+} \mathrm{N}$ electrostatic interactions. Regarding to $\mathbf{1 -}$ free $(\mathbf{H C l})$, the calculated $g_{\mathrm{Cl}-\mathrm{X}}$ profile reflects that the distance between the $\mathrm{Cl}^{-}$and the aromatics rings is slightly larger than for 1-3, reflecting that $\mathrm{Cl}^{-} \cdots \pi$ interactions are weaker in pure 5 aqueous solution than in aqueous acidic solution. However, the role of $\mathrm{Cl}^{-} \cdots \pi$ interactions is practically negligible in 1free $(\mathbf{H C l})$ since, as mentioned above, the number of anions is very low in the $\mathrm{HCl}$-free system.

On the other hand, the average number of the $\mathrm{H}_{3} \mathrm{O}^{+}$interacting 10 with the polymer chains (i.e. $\mathrm{H}_{3} \mathrm{O}^{+}$at $r<5 \AA$ ) is very small: 6 for $\mathbf{1}$ and 2 for $\mathbf{2}$ and $\mathbf{3}$. Moreover, the maximum number of $\mathrm{H}_{3} \mathrm{O}^{+}$ interacting with a polymer chain, which was 8 , was achieved for $\mathbf{1}$ while, in some snapshots, chains of $\mathbf{3}$ were not interacting with $\mathrm{H}_{3} \mathrm{O}^{+}$. The residence times of the $\mathrm{H}_{3} \mathrm{O}^{+}$were short, the maximum $15\left(\tau_{\max }\right)$ values reached for $\mathbf{1}, \mathbf{2}$ and $\mathbf{3}$ being $0.8,0.7$ and $0.4 \mathrm{~ns}$, respectively. These results suggest that the role of the $\mathrm{H}_{3} \mathrm{O}^{+}$in the gelation of the investigated ionenes is less relevant than that played by $\mathrm{Cl}^{-}$.

In general, results displayed in Figure 4 reflect a distinctive 20 internal hydration pattern for $\mathbf{1}$, which in turn promotes the gelation through the formation of appropriated environment...polymer interactions. More specifically, the presence of relatively small internal hydration regions that accommodate hydrated $\mathrm{Cl}^{-}$anions favours a gelation mechanism 25 in which such negatively charged species act as binders among polymer chains. More specifically, the presence of $\mathrm{Cl}^{-}$anions promote the formation of specific polymer $\cdots$ polymer interactions through a network of attractions and repulsions (e.g. $\mathrm{Cl}^{-} \ldots{ }^{+} \mathrm{N}$ and $\mathrm{Cl}^{-} \cdots \pi$, respectively), as is proved below. Simulations on 1${ }_{30}$ free $(\mathbf{H C l})$ demonstrate that $\mathrm{HCl}$ is required for the existence of such internal hydration nanoregions. These results successfully explain the drastic reduction in the gelation time observed for the ionene with an ortho topology when the neutral aqueous solution was acidified (Figure 2B).

35 Polymer $\cdots$ polymer interactions have been examined considering $\mathrm{N}-\mathrm{H} \cdots \mathrm{O}$ hydrogen bonds, $\mathrm{N}-\mathrm{H} \cdots \pi$, cation $\cdots \pi$ and $\pi-\pi$ stacking interactions. Intermolecular hydrogen bonds in 1-3 and 1-free(HCl), which have been examined through the radial distribution function for $\mathrm{H} \cdots \mathrm{O}$ pairs belonging to amide groups 40 of different chains ( $g_{\mathrm{H}-\mathrm{O}} ;$ Figure $\left.5 \mathrm{~A}\right)$, reveal important differences. More specifically, the $\mathrm{H} \cdots \mathrm{O}$ distance between hydrogen bonded amide groups is around $1.9 \AA$ for both $\mathbf{1}$ and $\mathbf{3}$, even though the population of this specific intermolecular interactions is several times greater for the former than for the 45 latter. This distance decreases slightly (i.e. to $\sim 1.8 \AA$ ) for $\mathbf{1 -}$ free $(\mathbf{H C l})$, reflecting also strong specific interactions between the polymer chains. For $\mathbf{2}$, the population of hydrogen bonds is intermediate between those of $\mathbf{1}$ and $\mathbf{3}$, even though the $\mathrm{H} \cdots \mathrm{O}$ distance increases and expands from 2.1 to $2.5 \AA$. These results 50 are fully consistent with FTIR observations for $\mathbf{1}$ in water and $\mathrm{HCl}$ environments, evidencing that the participation of the amide groups in hydrogen bonds is highly favoured for the system with an ortho substitution pattern. Figure $6 \mathrm{~A}$ reflects the crucial role played by intermolecular hydrogen bonds in the self-assembly 55 and, therefore, gelation properties, of $\mathbf{1}$.
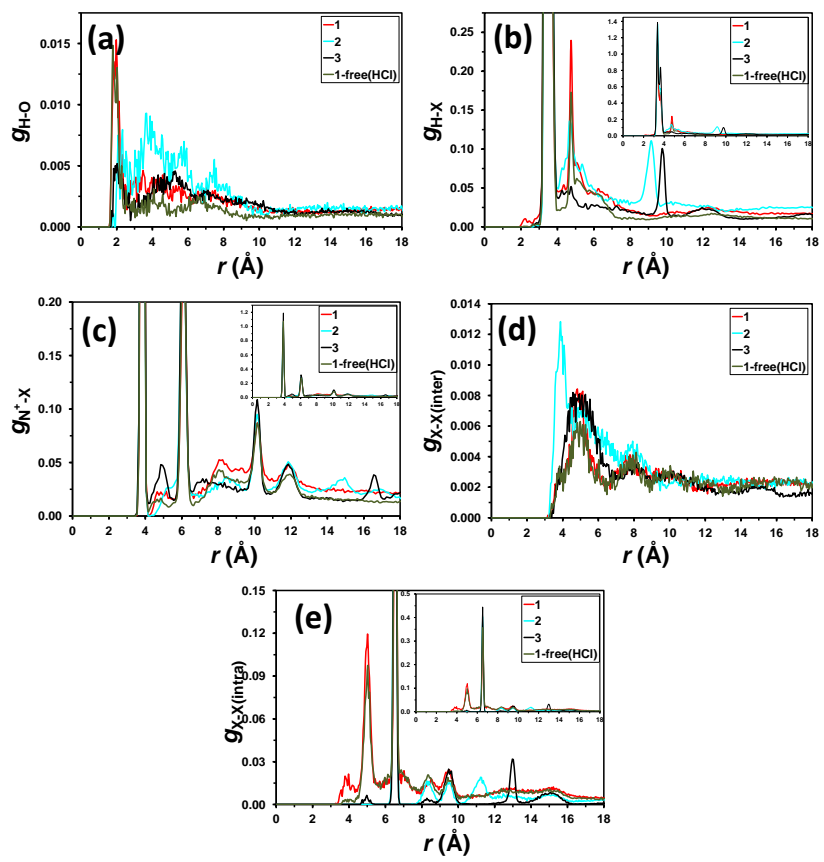

Fig. 5 Radial distribution functions for the A) $(\mathrm{N}-) \mathrm{H}^{\cdots} \mathrm{O}$ pairs belonging to different chains, B) $(\mathrm{N}-) \mathrm{H}^{\cdots} \mathrm{X}_{\mathrm{cm}}$ and $\left.\mathrm{C}\right) \mathrm{N}^{+} \cdots \mathrm{X}_{\mathrm{cm}}$ pairs belonging to 60 the same or different chains, and $\mathrm{X}_{\mathrm{cm}} \cdots \mathrm{X}_{\mathrm{cm}}$ pairs belonging to $\mathrm{D}$ ) different and $\mathrm{E}$ ) the same chains for 1-3 and 1-free $(\mathbf{H C l}) . \mathrm{X}_{\mathrm{cm}}$ refers to the centre of masses of the aromatic rings. Insets in B), C) and E) display the complete profiles with irrelevant peaks (i.e. those based on the molecular geometry rather than on the formation of interactions).

65 Both $\mathrm{N}-\mathrm{H} \cdots \pi$ and cation $\cdots \pi$ interactions have been examined by calculating the radial distribution functions for $(\mathrm{N}-) \mathrm{H} \cdots \mathrm{X}_{\mathrm{cm}}$ $\left(g_{\mathrm{H}-\mathrm{X}}\right)$ and $\mathrm{N}^{+} \ldots \mathrm{X}_{\mathrm{cm}}\left(g_{\mathrm{N}}{ }^{+} \mathrm{X}\right)$ pairs, respectively. The $g_{\mathrm{H}-\mathrm{X}}$ exhibits two sharp peaks centred at 3.3 and $\sim 3.6 \AA$ (Figure 5B, inset), which correspond to the intramolecular pairs involving the 70 aromatic rings directly linked to the $\mathrm{N}-\mathrm{H}$ and $\mathrm{C}=\mathrm{O}$ of the same amide group, respectively, the latter being the only influenced by the core topology. However, the sharp and broad peak for $\mathbf{1}$ and 2, respectively, at $\sim 4.7 \AA$ (Figure $5 \mathrm{~B}$ ) has been attributed to the formation of attractive interactions between amide groups and 75 aromatic rings belonging to different chains (Figure 6B). The geometry of this intermolecular interaction, which is poorly detectable for $\mathbf{3}$, is considerably better defined for $\mathbf{1}$ than for $\mathbf{2}$, even though the abundance is pretty similar for such two ionenes. Comparison of the peak at $\sim 4.7 \AA$ for $\mathbf{1}$ and $\mathbf{1 - f r e e}(\mathbf{H C l})$ indicates 80 that intermolecular $\mathrm{N}-\mathrm{H} \cdots \pi$ interactions are less abundant in pure aqueous solution than in acidic aqueous solution. This has been attributed to the noticeable role played by the $\mathrm{Cl}^{-}$anions in the latter that, as discussed above, surround the polymer chains and act as binders through the formation of abundant and strong 85 electrostatic $\mathrm{Cl}^{-} \ldots{ }^{+} \mathrm{N}$ interactions.

On the other hand, comparison of the ${g_{\mathrm{N}}}^{+} \mathrm{x}$ profiles and the different molecular topologies indicates that the sharp and intense peaks at $\sim 3.8, \sim 6.0$ and $\sim 10.1 \AA$ (Figure $5 \mathrm{C}$, inset) correspond to intramolecular pairs (i.e. $\mathrm{N}^{+}$atoms and the closer aromatic rings 90 in the same polymer chain). However, inspections of the peaks due to intermolecular cation $\cdots \pi$ interactions at $r \leq 5 \AA$ evidences that the amount of $\mathrm{N}^{+} \ldots \mathrm{X}_{\mathrm{cm}}$ pairs varies as $\mathbf{3}>\mathbf{1}>\mathbf{1 - f r e e}(\mathbf{H C l})>$ 2 (Figure 5C), even though it is relatively low in all cases. This 
result, which is fully consistent with our previous study, ${ }^{25}$ suggests that $\mathrm{N}^{+} \ldots \mathrm{X}_{\mathrm{cm}}$ are much less relevant for the gelation that hydrogen bonds and $\mathrm{N}-\mathrm{H} \cdots \pi$ interactions.

The formation of $\pi-\pi$ stacking interactions have been 5 examined considering separately the radial distribution of $\mathrm{X}_{\mathrm{cm}} \cdots \mathrm{X}_{\mathrm{cm}}$ pairs of aromatic rings belonging to different or the same chain $\left(g_{\mathrm{X}-\mathrm{X} \text { (inter) }}\right.$ and $g_{\mathrm{X}-\mathrm{X}(\mathrm{intra})}$, respectively). Inspection of the $g_{\mathrm{X}-\mathrm{X} \text { (inter) }}$ profiles (Figure 5D) reflects that, considering a threshold distance of $6 \AA$, the amount of intermolecular $\pi-\pi$ 10 stacking interactions decreases as follows: $2>\mathbf{3}>\mathbf{1}>\mathbf{1 -}$ free(HCl). The population of intermolecular $\pi-\pi$ stacking is $24 \%$, $40 \%$ and $50 \%$ lower for 3, 1 and $\mathbf{1 - f r e e}(\mathbf{H C l})$, respectively, than for 2). Moreover, the position of the first peak indicates that intermolecular $\pi-\pi$ stacking interactions are stronger for $\mathbf{2}$ than 15 for $\mathbf{1}, \mathbf{3}$ and $\mathbf{1 - f r e e}(\mathbf{H C l})$. Thus, the $g_{\mathrm{X} \text {-X(inter) }}$ profile calculated for 2 exhibits a high and sharp peak centred at $3.9 \AA$, which is very close to the optimum inter-ring distance predicted by quantum mechanical calculations for the strongest non-covalent interaction between $\pi$-systems. ${ }^{39}$ In contrast, 1, 3 and 1-free $(\mathbf{H C l})$ show a 20 broad and less intense peak centred at $\sim 4.9 \AA$, which is also identified as a local peak for 2 . Comparison of these results with experimental features and the interactions discussed above suggests that hydrophobically-driven intermolecular $\pi-\pi$ stacking interactions are not crucial for the gelation process in acid 25 environments. Thus, the entropic and entalphic contributions associated to the hydration of $\mathrm{Cl}^{-}$anions in small internal regions dominate with respect to hydrophobic effects in acid aqueous solutions. However, $\pi-\pi$ interactions play a key role in neutral water, where the strong inter-chain binding effects induced by the ${ }_{30} \mathrm{Cl}^{-}$anions are not present. ${ }^{25}$

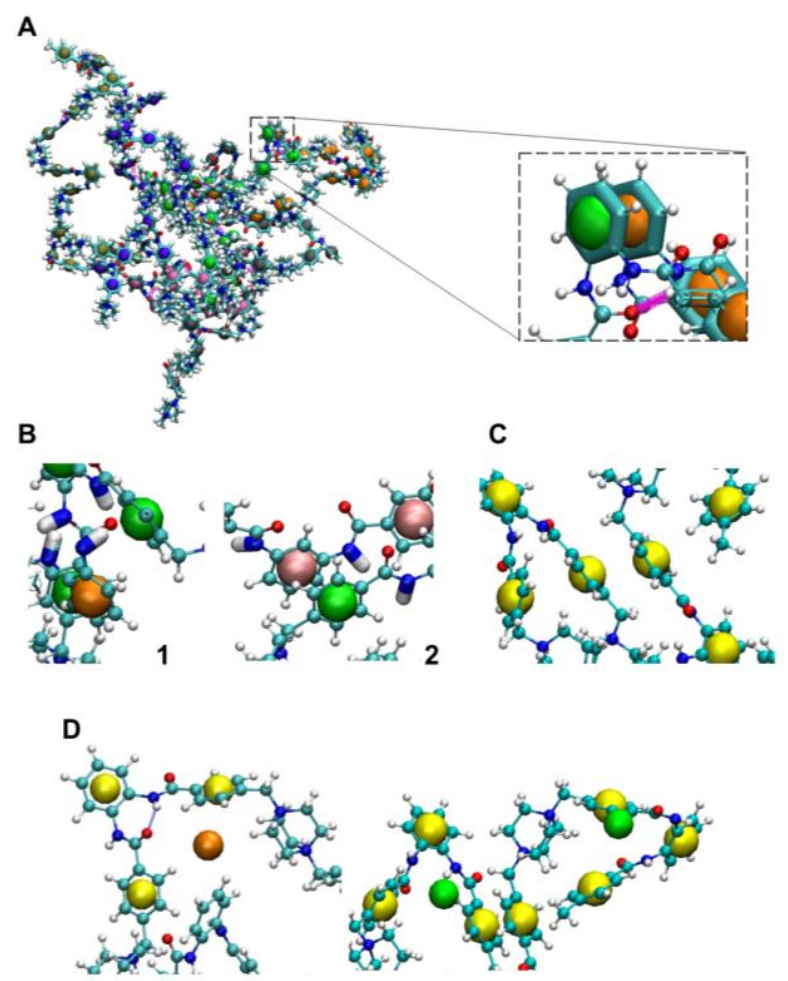

Fig. 6 A) Intermolecular hydrogen bonds, marked with pink dashed bars, in a representative snapshot of $\mathbf{1}$. The parameters used to define the 35 displayed hydrogen bonds were: $\mathrm{H} \cdots \mathrm{O} \leq 2.4 \AA$ (in agreement with the main peak observed in Figure 5A) and $\angle \mathrm{N}-\mathrm{H}^{\cdots} \mathrm{O}>100^{\circ}$. B) Representative intermolecular $\mathrm{N}-\mathrm{H} \cdots \pi$ interaction for $\mathbf{1}$ and 2. C) Representative intramolecular $\pi-\pi$ stacking network identified in 1. D) Snapshots illustrating the influence of the polymer $\cdots$ polymer $(l e f t)$ and ${ }_{40} \mathrm{Cl}^{-} \cdots$ polymer (right) interactions in the gelation of $\mathbf{1}$. Color codes: Carbon, nitrogen, oxygen and hydrogen atoms of the polymer chains are represented in light blue, dark blue, red and white, respectively. The electronic density of the aromatic rings has been represented using large balls centred in the phenyl groups, a colour being used for each polymer 45 chain (i.e. orange, green, yellow and pink for the first, second, third and fourth polymer chain). Finally, $\mathrm{Cl}^{-}$anions are represented by large independent (i.e. not centred in the aromatic rings) balls in $\mathrm{D}$.

The relative orientation between the interacting rings have been examined by analyzing the degree of tilting, which is 50 defined by the angle formed between the planes of the two rings, ( $\theta$ ) for all pairs of aromatic rings with at a $X_{\mathrm{cm}} \cdots \mathrm{X}_{\mathrm{cm}}$ distance lower than $6 \AA$, the latter value being derived from the $g_{\mathrm{X} \text {-X(inter) }}$ profiles (Figure 5D). The degree of tilting, which can range from $0^{\circ}$ to $90^{\circ}$, for two rings arranged in a sandwich or T-shaped 55 configuration (i.e. coplanar or perpendicular rings, respectively) is $\theta \leq 45^{\circ} \theta>45^{\circ}$. Results, which are represented in Figures 7A$\mathrm{B}$, indicate that, although both the sandwich and $\mathrm{T}$-shaped arrangements coexist, the former is the most abundant by around $3-10 \%$. Moreover, the distribution of the $\theta$ shows a broad peak 60 with a maximum at around $20^{\circ}$ for $\mathbf{1 - 3}$ and 1-free(HCl) (Figure 7B), reflecting a small distortion with respect to the ideal coplanar disposition of the interacting rings (i.e. an example of intermolecular $\pi-\pi$ stacking for $\mathbf{1}$ is displayed in Figure 6A).

The $g_{\mathrm{X}-\mathrm{X} \text { (intra) }}$ profiles (Figure $5 \mathrm{E}$ ) reflects the formation of ${ }_{65}$ intramolecular $\pi-\pi$ stacking interactions that are distinctive of $\mathbf{1}$ and to a lesser extend of 1-free $(\mathbf{H C l})$. More specifically, the broad and small peak centred at $\sim 3.8 \AA$ and more intense peak at $\sim 5.0 \AA$ have been associated to strong and weak intramolecular stacking interactions, respectively, whereas the sharp and intense 70 peak detected for the three ionenes at $6.5 \AA$ (Figure 5E, inset) corresponds to the neighbours non-interacting aromatic rings. Interestingly, although this kind of interaction is not detected for $\mathbf{2}$ and is scarce for $\mathbf{3}$ (i.e. the intramolecular interactions represents $2.5 \%$ of all $\pi-\pi$ stacking identified for 3 ), it is around $7525 \%$ more abundant than intermolecular $\pi-\pi$ stacking for both 1 and 1-free $(\mathbf{H C l})$ Moreover, analysis of the degree of tilting indicates that the $\mathrm{T}$-shaped is the most populated arrangement (Figures 7C), this tendency being more propounced for 1free $(\mathbf{H C l})$ than for $\mathbf{1}$. However, Figure 7D illustrates that there is 80 no well-defined peak in the corresponding distribution profiles. Indeed, inspection of a number of snapshots revealed the formation of intramolecular $\pi-\pi$ stacking networks in $\mathbf{1}$ and 1free $(\mathbf{H C l})$. This is illustrated for $\mathbf{1}$ in Figure $6 \mathrm{C}$, which shows a network of three interactions (two parallel and one T-shaped) 85 involving four rings of the same chain. These characteristics, which represent important differences with respect to the intermolecular $\pi-\pi$ stacking, reflects the importance of conformational flexibility of the polymer chains. Overall, the unique behaviour of $\mathbf{1}$ and $\mathbf{1 - f r e e}(\mathbf{H C l})$ in terms of intramolecular $90 \pi-\pi$ stacking suggests that these interactions play a crucial role in the gelation behaviour of the ionene with an ortho topology, independently of the $\mathrm{pH}$ of the aqueous solution 


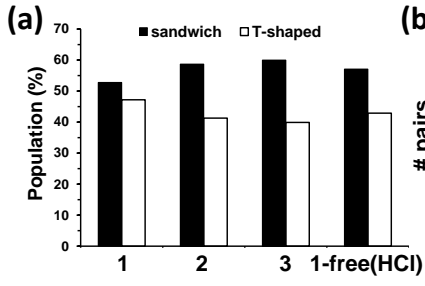

(b)

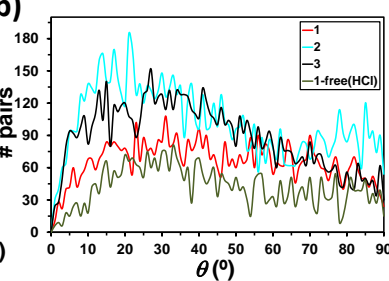

(c)

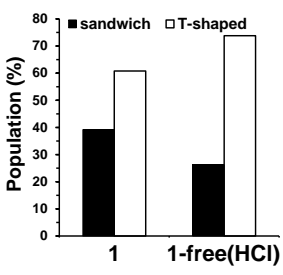

(d)

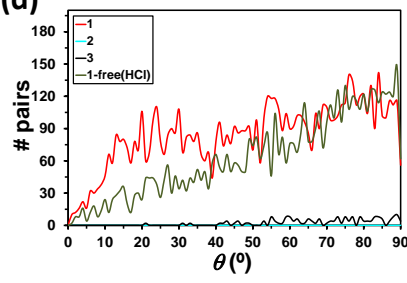

Fig. 7 For intermolecular A-B) and intermolecular C-D) $\pi-\pi$ stacking interactions: A-C) accumulative plots representing the population of 5 sandwich $\left(\theta \leq 45^{\circ}\right)$ and T-shaped $\left(\theta>45^{\circ}\right)$ arrangements for 1-3 and 1free(HCI); and B-D) distribution of the tilting angle $\theta$ for the accumulated pairs of aromatic rings at a distance smaller than $6 \AA$ for the last $1 \mathrm{~ns}$ of NPT-MD.

In summary, comparison of the information extracted MD 10 trajectories with the experimentally determined gelation capacity indicates that the interactions between the polymer chains and the acid counterion $\left(\mathrm{Cl}^{-}\right)$play a crucial role in the assembly process. More importantly, counterions affect polymer..polymer interactions, as is clearly depicted in Figure 6D. For example, 15 hydrogen bonding, $\mathrm{N}-\mathrm{H} \cdots \pi$ and intermolecular $\pi-\pi$ stacking, which are distinctive interactions in $\mathbf{1}$, are affected by the $\mathrm{Cl}^{-}$ located around the polymer chains. Indeed, $\mathrm{Cl}^{-}$anions coming from the acid solution are placed in the internal hydration areas surrounding polymer chains, and act as binders facilitating the 20 interactions among them. This mechanism is not possible in neutral aqueous solutions since the concentration of $\mathrm{Cl}^{-}$is too low. Polymer $\cdots$ polymer interactions are more abundant in $\mathbf{1}$ than in $\mathbf{2}$ and 3. Apparently, differences between the latter are much less clear. Thus, cation $\cdots \pi$ and intramolecular $\pi-\pi$ stacking 25 interactions are slightly preferred for 3 while $\mathrm{N}-\mathrm{H} \cdots \mathrm{O}$ and $\mathrm{N}-$ $\mathrm{H} \cdots \pi$, cation $\cdots \pi$ are slightly more favourable for 2 . However, analysis of the intermolecular $\pi-\pi$ stacking forces, which are considerably stronger and more abundant for $\mathbf{2}$ than for $\mathbf{3}$, suggests that these interactions are responsible for the 30 experimental observations. Thus, the remarkable differences found on the gelation of $\mathbf{2}$ and $\mathbf{3}$ should be attributed to the fact that the meta topology facilitates a greater approximation between aromatic rings of different molecules, which results in the formation of greater number of $\pi-\pi$ stacking interactions.

\section{${ }_{35}$ Conclusions}

The challenging gelation of acidic solutions was achieved using polycations bearing quaternary ammonium moieties. These ionene polymers were synthesized from a disubstituted phenylene dibenzamide core, which allows the construction of different 40 topomers (i.e. ortho-1, meta-2 and para-3). Remarkably, the topology of the polymers was found to play a crucial role on their aggregation behaviour both in pure water and in a variety of aqueous acidic solutions leading to the formation of uncommon acidic gels. In particular, ortho-1 showed superior gelation ability 45 than the analogues meta-2 and para-3 in solutions of different $\mathrm{pH}$ and ionic strengths. In terms of gel properties, lower critical gelation concentrations, higher gel-to-sol transition temperatures and faster gelation were routinely observed for ortho-1 topomer. Finally, atomistic MD simulations on huge models have allowed 50 to conclude that the distinctive properties of $\mathbf{1}$ are due to the

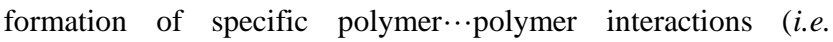
hydrogen bonds, $\mathrm{N}-\mathrm{H} \cdots \pi$ and intramolecular $\pi-\pi$ stacking), which are facilitated by the binder effect of $\mathrm{Cl}^{-}$counterions. Both the formation of intramolecular $\pi-\pi$ stacking networks and the 55 role of the $\mathrm{Cl}^{-}$in the stabilization of specific polymer $\cdots$ polymer interactions are singular trends with respect to $\mathbf{2}$ and $\mathbf{3}$. Thus, the ortho topology favours both the contact among polymer chains and, therefore, the formation of small internal hydration regions. This synergistic combination, which is not possible for the meta-2 60 and para-3, is responsible of the role played by $\mathrm{Cl}^{-}$counterions in the superior gelation of the former. Finally, differences between the two latter ionenes have been attributed to the greater strength of intermolecular $\pi-\pi$ stacking in 2. Future experiments involving dynamic light scattering and ultrasonic attenuation spectroscopy 65 to understand the topological influence of the hydrodynamic radius in solution approaching the CGC are currently underway in our laboratories.

\section{Acknowledgements}

This work was supported by the University of Regensburg, the 70 Deutsche Forschungsgemeinschaft (DFG) (1748/3-1; 1748/3-2), Ministerio de Economía y Competitividad (MINECO) and European FEDER funds (MAT2015-69367-R). We thank M. Häring and M. Tautz (Universität Regensburg) for crosschecking the formation of some gels, and the group of Prof. A. Göpferich 75 (Universität Regensburg) for kindly allowing us to use the rheometer. C.A. is grateful to ICREA Academia program. D.D.D. thanks the DFG for the Heisenberg Professorship Award.

\section{Notes and references}

1 R. R. Netz and D. Andelman, Physics Reports, 2003, 380, 1-95.

802 A. Y. Grosberg, T. T. Nguyen and B. I. Shklovskii, Rev. Mod. Phys., 2002, 74, 329-345.

3 C. Poinsignon, Mat. Sci. Eng., B, 1989, B3, 31-37.

4 W. Jaeger, J. Bohrisch and A. Laschewsky, Prog. Polym. Sci., 2010, 35, 511-577.

855 C. Werner, Advances in Polymer Science: Polymers for Regenerative Medicine. Springer: Dresden, 2006.

6 S. Punyani and H. Singh, J. Appl. Polym. Sci., 2006, 102, 1038-1044.

7 H. Kourai, T. Yabuhara, A. Shirai, T. Maeda and H. Nagamune, Eur. J. Med. Chem., 2006, 41, 437-444.

908 A. N. Zelinkin, D. Putnam, P. Shastri, R. Langer and V. A. Izumrudov, Bioconjugate Chem., 2002, 13, 548-553.

9 E. Bortel, A. Kochanowski, B. Siniarska and E. Witek, Polish J. Appl. Chem., 2001, 44, 54-78.

10 H. Noguchi, in Polymeric materials encyclopedia, J. C. Salomone,

95 (Ed.); CRC Press: Boca Raton, London, New York, Tokyo, 1996, pp $3392-3421$.

11 A. Laschewsky, Curr. Opin. Colloid Interface Sci., 2012, 17, 56-63.

12 S. R. Williams and T. E. Long, Prog. Polym. Sci., 2009, 34, 762-782.

13 E. R. Littmann and C. S. Marvel, J. Am. Chem. Soc., 1930, 52, $287-$ 100294. 
14 C. F. Gibss, E. R. Littmann and C. S. Marvel, J. Am. Chem. Soc., 1933, 55, 753-757.

15 M. Friedman, J. Agric. Food Chem., 2003, 51, 4504-4526.

16 J. K. Oha, R. Drumright, D. J. Siegwart and K. Matyjaszewski, Prog. Polym. Sci., 2008, 33, 448-477.

17 M. Yoshida, Synthesiology, 2012, 5, 181-189.

18 S. Schlick, Ionomers: Characterization, Theory, and Applications. CRC Press: Boca Raton, 1996.

19 M. R. Tant, K. A. Mauritz, and G. L. Wilkes, Ionomers: Synthesis,

10 Structure, Properties, and Applications. Blackie Academic and Professional: London, 1997.

20 A. Kowalenko, A. E. Kobryn, S. Gusarov, O. Lyubimova, X. Liu, N. Blinov and M. Yoshida, Soft Matter, 2012, 8, 1508-1520.

21 Y. He, H.-K. Tsao and S. Jiang, J. Phys. Chem. B, 2012, 116, 5766-

155770.

22 S. G. Lee, G. F. Brunello, S. S. Jang and D. G. Bucknall, Biomaterials, 2009, 30, 6130-6141.

23 S. S. Jang, W. A. Goddard, M. Y. S. Kalani, D. Myung and C. W. Frank, J. Phys. Chem. B, 2007, 111, 14440-14440.

2024 E. Chiessi, F. Cavalieri and G. Paradossi, J. Phys. Chem. B, 2007, 111, 2820-2827.

25 J. Bachl, D. Zanuy, D. E. López-Pérez, G. Revilla-López, C. Cativiela, C. Alemán and D. D. Díaz, Adv. Funct. Mater., 2014, 24, 4893-4904.

2526 Y. Misawa, N. Koumura, H. Matsumoto, N. Tamaoki and M. Yoshida, Macromolecules, 2008, 41, 8841-8846.

27 T. Takewaki, L. W. Beck and M. E. Davis, Micropor. Mesopor. Mat., 1999, 33, 197-207.

28 H. Omidian and J. G. Rocca, U.S. Pat. Appl. Publ., 2008, US

$30 \quad 20080089940$ A1 20080417.

29 J.-J. Xuan, Y.-D. Yan, D. H. Oh, Y. K. Choi, C. S. Yong and H.-G. Choi, Drug. Deliv., 2011, 18, 305-311.

30 M. J. Molina, M. R. Gómez-Antón, B. L. Rivas, H. A. Maturana and I. F. Piérola, J. Appl. Polym. Sci., 2001, 79, 1467-1475.

3531 M. Suzuki, S. Owa, M. Yumoto, M. Kimura, H. Shirai and K. Hanabusa, Tetrahedron Lett., 2004, 45, 5399-5402.

32 M. Suzuki, M. Yumoto, H. Shirai and K. Hanabusa, Org. Biomol. Chem., 2005, 3, 3073-3078.

33 M. Suzuki, M. Yumoto, M. Kimura, H. Shirai and K. Hanabusa, 40 Tetrahedron Lett., 2004, 45, 2947-2950.

34 M. Yamanaka and D. Higashi, PCT Int. Appl., 2012, WO 2012121394 A1 20120913.

35 I. Hwang, W. S. Jeon, H.-J. Kim, D. Kim, H. Kim, N. Selvapalam, N. Fujita, N. Shinkai and K. Kim, Angew. Chem. Int. Ed., 2007, 46, $45 \quad 210-213$.

36 S. W. Jeong and S. Shinkai, Nanotechnology, 1997, 8, 179-183.

37 J. E. Eldrige and J. D. Ferry, J. Phys. Chem., 1954, 58, 992-995.

38 H. Takayasu, Fractals in the Physical Sciences; Manchester University Press: Manchester and New York, 1990; pp 11-17.

5039 F. Rodríguez-Ropero, D. Zanuy and C. Alemán, J. Comput. Chem., 2008, 29, 69-78. 